\title{
A transmembrane inner nuclear membrane protein in the mitotic spindle
}

Ricardo Figueroa, ${ }^{1,2}$ Santhosh Gudise, ${ }^{1,3}$ Veronica Larsson ${ }^{4}$ and Einar Hallberg ${ }^{1, *}$

${ }^{1}$ Life Sciences; Södertörns University; Huddinge, Sweden; ${ }^{2}$ Department of Biochemistry and Biophysics; ${ }^{3}$ Department of Biosciences and Nutrition; Karolinska Institutet; Huddinge, Sweden; ${ }^{4}$ Department of Neurochemistry; Stockholm University; Stockholm, Sweden

Key words: inner nuclear membrane, transmembrane proteins, mitotic spindle, spindle matrix, mitosis, cancer, LINC complex, centrosome, dystonia

Submitted: 01/29/10

Revised: 02/18/10

Accepted: 02/18/10

Previously published online: www.landesbioscience.com/journals/ nucleus/article/11738

*Correspondence to:

Einar Hallberg; Email: einar.hallberg@sh.se
$\mathrm{W}$ have recently characterized a novel transmembrane protein of the inner nuclear membrane of mammalian cells. The protein has two very interesting features. First, despite being an integral membrane protein it is able to concentrate in the membranes colocalizing with the mitotic spindle in metaphase and anaphase. Hence, the protein was named Samp1, Spindle associated membrane protein 1 . Secondly, it displays a functional connection to centrosomes. This article discusses various aspects of Samp1 in relation to possible cellular function(s).

\section{Introduction}

The nuclear envelope (NE), separating the nucleoplasm from the cytoplasm, consists of two concentric lipid membranes, the nuclear pores and the nuclear lamina. ${ }^{1,2}$ The inner nuclear membrane (INM) has recently attracted increased attention. This is due to the discovery of a link between a group of diverse diseases, called "laminopathies" or "envelopathies", and mutations in genes encoding proteins of the nuclear envelope (NE), e.g., the lamins and INM proteins. Intensified research on the pathogenesis of these diseases has revealed new and unexpected roles of the INM and the nuclear lamina in diverse vital cellular functions including cell signaling, chromosome organization and gene regulation. ${ }^{3-5}$

In rat liver the $\mathrm{NE}$ has been estimated to contain about 80 unique integral membrane proteins, ${ }^{6}$ most of which the function and characteristic are still unknown.
Recently, we have characterized one of these putative NE proteins in human cells and showed that it is indeed an integral membrane protein specifically located in the INM. ${ }^{7}$ One striking property of this protein is its distribution in membranes along mitotic spindle microtubules (Fig. 1A), leading us to name it Samp1 (Spindle associated membrane protein 1). Another interesting feature is its functional association with centrosomes (Fig. 1B).

\section{Membranes in the Mitotic Spindle}

In our recent paper ${ }^{7}$ we showed that in mitotic HeLa, MDCK and neuroblastoma cells Samp1 colocalized with microtubules of the mitotic spindle and the membrane marker $\mathrm{DiOC}_{6}$. This is the first demonstration in intact cells of the existence of a distinct membrane sub-domain in the mitotic spindle containing at least one integral membrane protein, Samp1. The finding was surprising because INM proteins are generally known to be dispersed in the contiguous mitotic ER and to be recruited back to reforming daughter nuclei only after chromosome segregation occurring from anaphase through $\mathrm{G}_{1}{ }^{8}$ Supporting our observation, a more recent study by $\mathrm{Lu}$ and colleagues presents high resolution deconvolved spherical aberration corrected confocal images clearly showing the distribution of GFP-tagged ER proteins along kinetochore microtubules.9 Previously, B-type lamins have been reported to locate in mitotic spindles of HeLa cells. ${ }^{10}$ Although B-type lamins are not integral membrane proteins they are able to associate to membranes by 


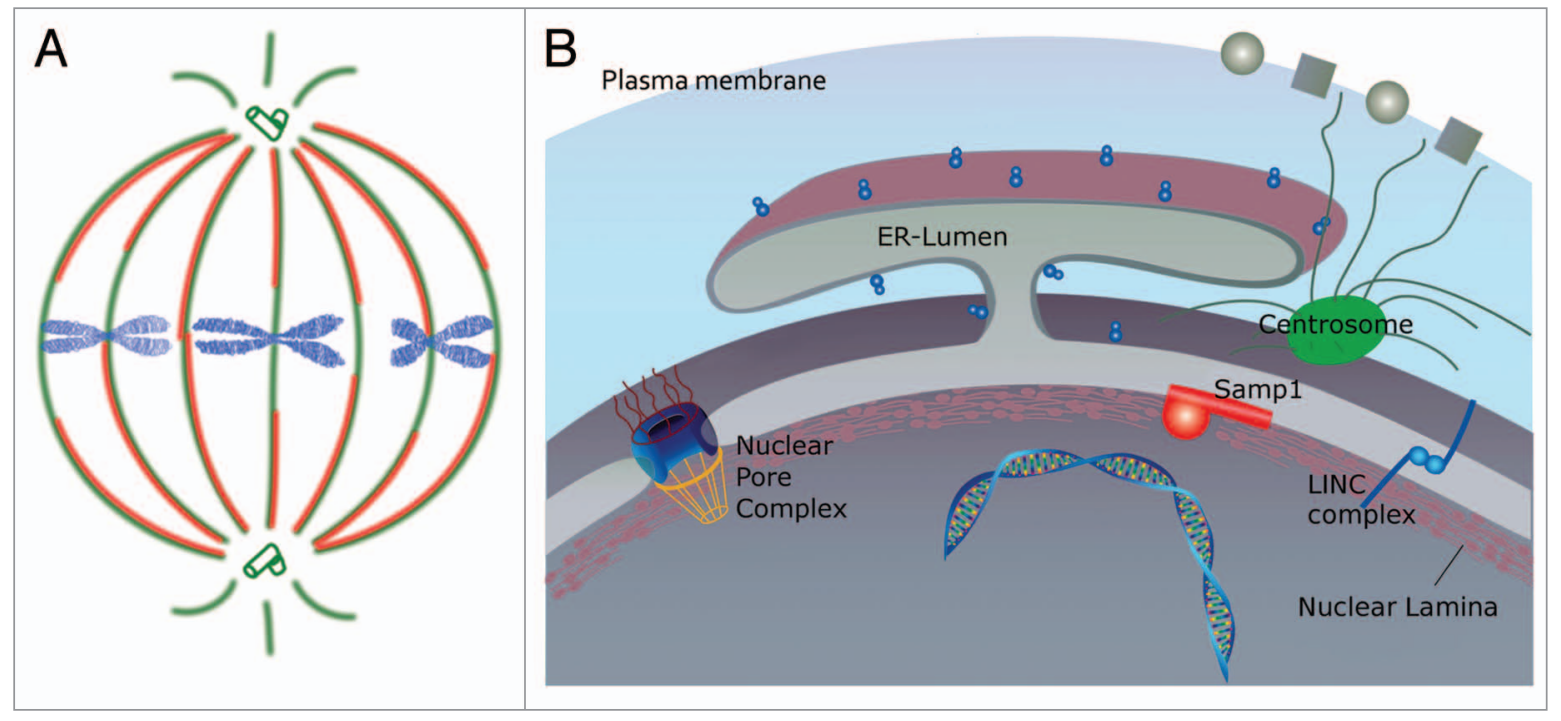

Figure 1. Schematic illustrations of the distribution of Samp1 in interphase and mitosis. (A) In mitosis a distinct membrane sub-domain (red) containing Samp1 extends along mitotic microtubuli (green), which align chromosomes (blue) in the metaphase plate. (B) In interphase Samp1 is located in the INM where it may connect to the cytoskeleton via the LINC complex.

virtue of the covalently linked farnesyl group in their carboxy-termini and by binding to integral membrane proteins. This membrane sub-domain may be a new component of the mysterious "spindle matrix" reviewed in reference 11 .

In the study by $\mathrm{Lu}$ and colleagues the membrane tubules observed along kinetochore microtubules were believed to be continuous with the more peripheral mitotic ER and estimated to contain approximately $0.1-0.2 \%$ of the mitotic ER.' One important distinction from bulk ER proteins however, is that Samp1 is the only transmembrane protein discovered so far to concentrate in metaphase and anaphase spindles, ${ }^{7}$ suggesting an active role for Samp1 and membranes in the mitotic process. This idea gains support from a genome wide screen of $C$. elegans embryonal development using DIC time-lapse microscopy. Early embryos from eggs of worms that had been microinjected with siRNA directed against the Samp1 homolog displayed a defective pronuclear and nuclear appearance, a phenotype often accompanied by mitotic spindle defects. ${ }^{12}$ If Samp1 proves to have a mitotic function it may be the first transmembrane protein described to play a role in the mitotic machinery. Recently, a group of peripheral proteins from the NPC and proteins involved in nucleocytoplasmic transport have been assigned important functions as spindle assembly factors and checkpoint proteins. ${ }^{13-19}$ In the future it will be exciting to determine if Samp1 and perhaps other INM proteins can be added to the growing list of nuclear proteins with double duties required for orchestrating the open mitotic process. ${ }^{20}$

\section{Is Samp1 a Novel Component of the LINC Complex?}

Quite unexpectedly with respect to its location in the INM, Sampl was found to be functionally connected to the centrosomes in the cytoplasm. This was evidenced by siRNA mediated posttranscriptional silencing which resulted in detachment of centrosomes from the NE. Interestingly, detached centrosomes are also found in embryonic fibroblasts from double knock-out lamin A/C $\left(\right.$ lmna $\left.^{-1}\right)$ mice, ${ }^{21}$ in fibroblasts from X-linked EmeryDreifuss muscular dystrophy patients that lack a functional $E M D$ gene encoding the INM protein emerin or in normal fibroblasts after posttranscriptional silencing of emerin. ${ }^{22}$ Because emerin interacts with lamin A and is dependent on lamin A for its INM localization, the observed phenotype (centrosomal detachment) likely is a reflection of a malfunctioning LINC (Linker of Nucleoskeleton and Cytoskeleton) complex. ${ }^{3,5,23}$ The LINC complex connects the cytoskeleton with the chromatin in the nucleoplasm via a trans-cisternal network of interactions including transmembrane $\mathrm{KASH}$ proteins in the outer nuclear membrane (ONM) of the NE and SUN proteins in the INM. The LINC complex is conserved from yeast to mammals and has been found to play essential roles in many vital cell functions including cell polarization, nuclear migration and positioning, cell signaling, chromosomal movement, cell division and meiosis. The two INM components of the LINC complex, Sun1 and Sun2, were recently shown to play critical roles in anchoring myocytic synaptic nuclei close to the neuromuscular junction, ${ }^{23,24}$ highlighting the importance of the LINC complex for nuclear movement and positioning. The LINC complex has even been suggested to mediate mechanotransduction from the cell surface to the nuclear interior affecting chromatin organization and gene regulation..$^{25}$ Interestingly, Ima1 (the $S$. pombe homologue of Samp1) was shown to play a central role in mediating and buffering mechanical forces from the microtubule cytoskeleton via SUN and KASH homologues to centromeric heterochromatin of fission yeast. ${ }^{26}$ This function of Ima1 together with the functional association between Samp1 and centrosomes observed by us, ${ }^{7}$ suggest that Samp1 may be a component of the LINC complex in mammalian cells. 
Figure 2. Expression profile of Samp1. (A) Relative transcription profiles of Samp1 (green) and Lamin B1 (blue) in adult and developing mice. Note the high mRNA expression levels in oocytes $\left(p<3.49 \times 10^{-9}\right)$ and fertilized eggs $\left(p<6.1 \times 10^{-10}\right)$.

(B) Heat map representation of the relative expression of Samp1 or Lamin B1 during early development in the mouse. The relative intensity is color coded according to the scale on the right. Data collected from Array Express or GEO databases as indicated on the left. The average of the different profiles were calculated and displayed at the bottom. (C) Heat map showing the effect of cell proliferation on expression of Samp1 and lamin B1 in human primary fibroblasts. Relative gene expression profiles from GEO (indicated on the left) color coded as in (A).
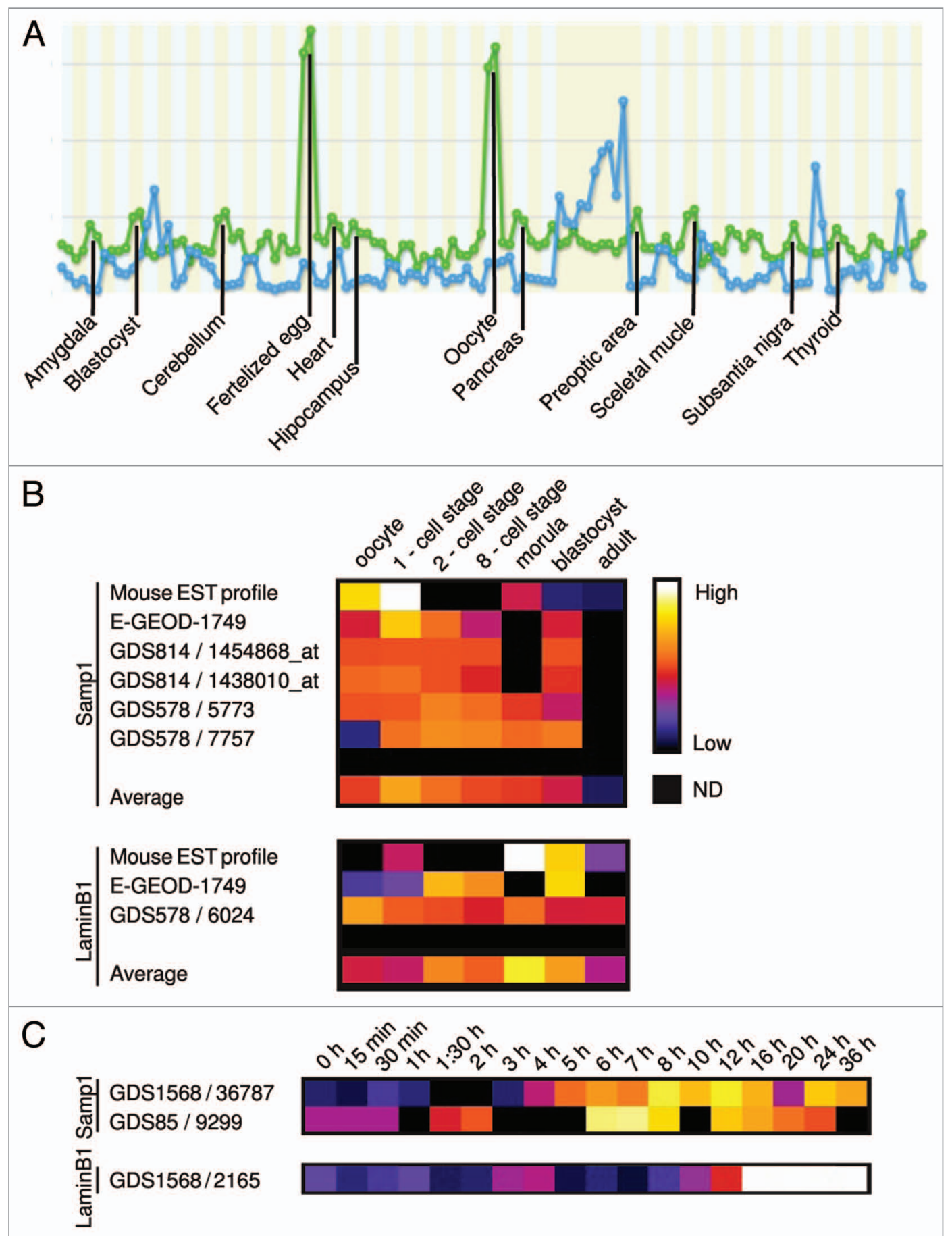

\section{Chromosomal Position and Expression of Samp1}

The chromosomal location of the samp1 gene is at $1 \mathrm{p} 36.22$, an area, which has been genetically linked to a rare autosomal dominant disorder of the central nervous system, dystonina 13 (DYT13). DYT13 is characterized by involuntary muscle contractions in the cranial and cervical region or in the upper limbs, leading to repetitive twisting movements. ${ }^{27-29}$ Compelling recent studies suggest that another form of primary dystonia, DYT1, is associated with a dysfunctional NE. In view of these findings the Sampl gene is an interesting candidate in search of a mechanism behind DYT13 dystonia.

In search of a function for Samp1 we have compiled information from databases and the literature. Analysis of tissue specific distribution of Samp1 mRNA expression showed very high levels in the oocyte and the fertilized egg (Fig. 2A). Moderately high levels were found in a number of tissues including the cerebellum and skeletal muscle. The expression profile for lamin B1, a constitutively expressed marker of the NE was dramatically different, suggesting that the expression of Samp1 is not simply following the number or size of the nuclei in different 


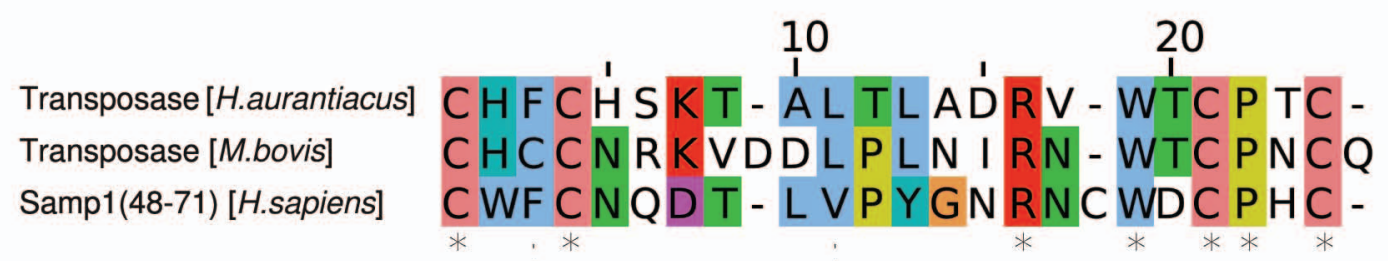

Figure 3. Sequence similarity between Samp1 and DNA binding proteins. A segment over the two upstream-CXXC-motifs corresponding to amino acids 48-71 of human Samp1 displays 39\% sequence similarity with two transposases (IS605 family transposase OrfB, GENE ID: 5735257 Haur_3396) from Herpetosiphon aurantiacus and (GENE ID: 6390628 orf19) from Moraxella bovis. The two downstream—CXXC—motifs only display gene specific homology in other species.

tissues. Compilations of expression profiles of Samp1 during early development show that Samp1 is highly expressed during oogenesis (not shown) and peaking at the one-cell and two-cell stages after fertilization (Fig. 2B). This is interesting in view of the small pronuclei phenotype observed in C. elegans embryos (see above), ${ }^{12}$ suggesting that Samp1 indeed may have a function during early development. In S. pombe, the Samp1 homologue Imal is not essential for viability, but knockout strains had a six-fold longer generation time. ${ }^{26}$ This prompted us to search databases for Sampl expression in relation to cell proliferation. After starvation of tissue culture cells Sampl expression displayed a dramatic increase already 5 hours after the addition of serum and peaked at $8-12$ hours (Fig. 2C). This was much earlier compared to lamin B1 expression, which increased dramatically only after 12-16 hours, suggesting that Samp1 may be important during the $S$-phase. ${ }^{30}$

\section{Structural Aspects}

The N-terminal half of Samp1 constitutes a functional domain essential for targeting to the INM. ${ }^{7}$ It is also the structurally most exciting part of Samp1, containing four tetra-amino acid-CXXCmotifs, which are highly conserved from S. pombe to H. sapiens. ${ }^{7.26}$ These motifs could potentially form two Zinc fingers, where either or both may interact with proteins and/or nucleic acids. In support of Zinc finger(s) in Samp1 is the requirement for bivalent cations for the stability of a purified recombinant fragment corresponding to this domain (preliminary results). A 23 amino acids stretch containing the first two-CXXC-motifs of human Samp1 show 39\% sequence similarity with two prokaryotic transposases (Fig. 3). Transposases require direct binding to DNA for their function. ${ }^{31,32}$ However, the ability of Samp1 to bind nucleic acids remains to be determined.

\section{Concluding Remarks}

Future studies of this interesting protein may reveal new and unexpected functions of membranes and bi-functional nuclear proteins in the mitotic spindle and also enlighten our understanding of the many vital functions of LINC complexes, a rapidly growing research field.

\section{Acknowledgements}

This work was supported by grants from the Swedish Research council (\#20065977 and \#2006-3468), the Swedish Cancer Society (\#07 0093) and by Magnus Bergvalls stiftelse.

\section{References}

1. Hetzer MW, Walther TC, Mattaj IW. Pushing the envelope: structure, function, and dynamics of the nuclear periphery. Annu Rev Cell Dev Biol 2005; 21:347-80

2. Stewart CL, Roux KJ, Burke B. Blurring the boundary: the nuclear envelope extends its reach. Science 2007; 318:1408-12.

3. Dauer WT, Worman HJ. The nuclear envelope as a signaling node in development and disease. Dev Cell 2009; 17:626-38

4. Worman HJ, Bonne G. "Laminopathies": a wide spectrum of human diseases. Exp Cell Res 2007; 313:2121-33

5. Mèjat A, Misteli T. LINC complexes in health and disease. Nucleus 2010; 1.

6. Schirmer EC, Florens L, Guan T, Yates JR, 3rd, Gerace L. Nuclear membrane proteins with potential disease links found by subtractive proteomics. Science 2003; 301:1380-2.

7. Buch C, Lindberg R, Figueroa R, Gudise S, Onischenko E, Hallberg E. An integral protein of the inner nuclear membrane localizes to the mitotic spindle in mammalian cells. J Cell Sci 2009; 122:2100-7.
8. Dultz E, Zanin E, Wurzenberger C, Braun M, Rabut G, Sironi L, et al. Systematic kinetic analysis of mitotic dis- and reassembly of the nuclear pore in living cells. J Cell Biol 2008; 180:857-65.

9. Lu L, Ladinsky MS, Kirchhausen T. Cisternal organization of the endoplasmic reticulum during mitosis. Mol Biol Cell 2009; 20:3471-80.

10. Tsai MY, Wang S, Heidinger JM, Shumaker DK, Adam SA, Goldman RD, et al. A mitotic lamin B matrix induced by RanGTP required for spindle assembly. Science 2006; 311:1887-93.

11. Johansen KM, Johansen J. Cell and molecular biology of the spindle matrix. International review of cytology 2007; 263:155-206.

12. Sonnichsen B, Koski LB, Walsh A, Marschall P, Neumann B, Brehm M, et al. Full-genome RNAi profiling of early embryogenesis in Caenorhabditis elegans. Nature 2005; 434:462-9.

13. Harel A, Forbes DJ. Importin beta: conducting a much larger cellular symphony. Mol Cell 2004; 16:319-30.

14. Blower MD, Nachury M, Heald R, Weis K. A Rae1containing ribonucleoprotein complex is required for mitotic spindle assembly. Cell 2005; 121:223-34.

15. Zuccolo M, Alves A, Galy V, Bolhy S, Formstecher E, Racine V, et al. The human Nup107-160 nuclear pore subcomplex contributes to proper kinetochore functions. EMBO J 2007; 26:1853-64.

16. Lince-Faria M, Maffini S, Orr B, Ding Y, Claudia F, Sunkel CE, et al. Spatiotemporal control of mitosis by the conserved spindle matrix protein Megator. J Cell Biol 2009; 184:647-57.

17. Orjalo AV, Arnaoutov A, Shen Z, Boyarchuk Y, Zeitlin SG, Fontoura B, et al. The Nup107-160 nucleoporin complex is required for correct bipolar spindle assembly. Mol Biol Cell 2006; 17:3806-18.

18. Joseph J, Liu ST, Jablonski SA, Yen TJ, Dasso M. The RanGAP1-RanBP2 complex is essential for microtubule-kinetochore interactions in vivo. Curr Biol 2004; 14:611-7.

19. Mishra RK, Chakraborty P, Arnaoutov A, Fontoura BM, Dasso M. The Nup107-160 complex and gamma-TuRC regulate microtubule polymerization at kinetochores. Nat Cell Biol 2010.

20. De Souza CP, Osmani SA. Double duty for nuclear proteins-the price of more open forms of mitosis. Trends Genet 2009; 25:545-54.

21. Hale CM, Shrestha AL, Khatau SB, StewartHutchinson PJ, Hernandez L, Stewart CL, et al. Dysfunctional connections between the nucleus and the actin and microtubule networks in laminopathic models. Biophys J 2008; 95:5462-75.

22. Salpingidou G, Smertenko A, HausmanowaPetrucewicz I, Hussey PJ, Hutchison CJ. A novel role for the nuclear membrane protein emerin in association of the centrosome to the outer nuclear membrane. J Cell Biol 2007; 178:897-904.

23. Starr DA. A nuclear-envelope bridge positions nuclei and moves chromosomes. J Cell Sci 2009; 122:577-86. 
24. Lei K, Zhang X, Ding X, Guo X, Chen M, Zhu B, et al. SUN1 and SUN2 play critical but partially redundant roles in anchoring nuclei in skeletal muscle cells in mice. Proc Natl Acad Sci USA 2009; 106:1020712.

25. Wang N, Tytell JD, Ingber DE. Mechanotransduction at a distance: mechanically coupling the extracellular matrix with the nucleus. Nat Rev Mol Cell Biol 2009; 10:75-82.

26. King MC, Drivas TG, Blobel G. A network of nuclear envelope membrane proteins linking centromeres to microtubules. Cell 2008; 134:427-38.

27. Tanabe LM, Kim CE, Alagem N, Dauer WT. Primary dystonia: molecules and mechanisms. Nat Rev Neurol 2009; 5:598-609.
28. Bentivoglio AR, Ialongo T, Contarino MF, Valente EM, Albanese A. Phenotypic characterization of DYT13 primary torsion dystonia. Mov Disord 2004; 19:200-6.

29. Brancati F, Defazio G, Caputo V, Valente EM, Pizzuti A, Livrea P, et al. Novel Italian family supports clini$\mathrm{cal}$ and genetic heterogeneity of primary adult-onset torsion dystonia. Mov Disord 2002; 17:392-7.

30. Iyer VR, Eisen MB, Ross DT, Schuler G, Moore T, Lee JC, et al. The transcriptional program in the response of human fibroblasts to serum. Science $1999 ; 283: 83-7$.
31. Tobiason DM, Lenich AG, Glasgow AC. Multiple DNA binding activities of the novel site-specific recombinase, Piv, from Moraxella lacunata. J Biol Chem 1999; 274:9698-706.

32. Barabas O, Ronning DR, Guynet C, Hickman AB, Ton-Hoang B, Chandler $M$, et al. Mechanism of IS200/IS605 family DNA transposases: activation and transposon-directed target site selection. Cell 2008; 132:208-20. 